Technical progress to date (July 31,1991 - July 31,1992$)$

I. utroduction.

The objective of this project is to develop a time-of-flight mass spectrometric approach to ordering Sanger sequence fragments, replacing electrophoresis and removing the electrophoresis bottleneck to rapid DNA sequencing. When the project was funded, we had demonstrated that massive DNA molecules could be volatilized, substantially intact, by a process involving pulsed laser ablation of a froz $n$ film of a DNA solution. Using a crude time-of-flight mass spectrometer, we had demonstrated that ions of the ablated DNA could be formed, and that mass spectra were obtainable which appeared to contain only the parent molecular ion. The laser used was a dye laser which we tuned to match sodium atom resonances to increase the ionization efficiency. At about the same time, others were developing comparable techniques for mass spectrometry of proteins; the proteins were volatilized and ionized by UV laser irradiation of a variety of solid organic acids cocrystallized with the protein. Preliminary experiments suggested that this approach worked very poorly for DNA, but the simplicity of sample preparation (dry vs. frozen liquid) impelled us to investigate this approach also.

\title{
II. Time-of-flight mass spectrometry
}

Construction of the time-of-flight mass spectrometer was described in the 1991 report. The system has wc. ked well, operating up at sample potentials up to $40 \mathrm{kV}$ with samples at liquid nitrogen temperature (high accelerating voltages aid detection of massive molecular ions). We have used the system to continue to search for optimum ways of generating intact molecular ions of DNA, and have also worked with protein samples, fullerenes and some unique phthalocyanine polymers developed at ASU, because these different samples offer insight into aspects of instrument performance and the physics of ablation of intact massive molecular ions.

Work towards DNA mass spectrometry has taken three directions.

\section{II.a Ablation/ionization of DNA from ice films}

This process was demonstrated in our preliminary work prior to funding and optimization and reduction of the technique to a routine procedure has been the major goal of our work this past year. As originally developed, the process appeared to be controlled by three key parameters. First, ice films needed to be sufficiently thin (on the order of a few micrometers) to be completely vaporized by a shock wave driven by laser vaporization of a surface layes of the copper substrate. The state of the copper substrate surface appeared to be important, corroded surfaces giving less fragmentation in our collector experiments and somewhat more reproducible mass spectra, presumably due to better absorption properties. Finally, ionization appeared to be more efficient and reproducible 
when the ablating laser was tuned to a sodium atom transition; arguably, this produced sodium ions which could attach to the ablated DNA molecules. We have spent the past year attempting to understand, control ano optimize these processes, and searching for alternate routes to ablation and ionization which offer better controllability/reproducibility and better potential compatibility with the high-throughput operation necessary for largescale DNA sequencing.

To achieve the thickness required for complete ablation, frozen films were initially allowed to sublime during pumpdown in a rather poorly controlled way, and the film area of uneven thickness was searched for a region with the right combination of properties to yield the desired molecular ions. It was not unusual to search over an area up to $1 \mathrm{~cm}^{2}$ in order to find a region perhaps less than $1 \mathrm{~mm}^{2}$ which gave a good mass spectrum. We have worked to improve this approach in several ways. We built an ancillary sublimator, with a small glass bell jar which allows good visual observation of the subliming film and can allow observation of interference fringes from thin films on polished substrates. With this it has become fairly routine to obtain films that appear to the eye to have sizeable areas of a suitable thickness. We have also built a simple spin-cast system, and with it can produce controllable spun films a few thousand angstroms thick. A major goal of our work has been to develop sample preparation techniques that do not involve the omplication of freezing. We have discovered that solutions of tris-EDTA buffer, when sufficiently concentrated ( $\sim \mathrm{M}$ tris $/ 0.1 \mathrm{M}$ EDTA), do not evaporate to dryness in vacuum, but become viscous, almost glassy, highly-concentrated liquid films. By controlled deposition and vacuum evaporation from polished substrates it is possible to prociuce such films with thickness ranges measurable by interference fringes ranging from a few thousand angstroms to a few micrometers. These films can be cleanly ablated by laser pulses of sufficient power. Our hope with such liquid, room temperature films is not only to achieve simple sample preparation but also to avoid concerns about solute segregation that arise when DNA solutions are frozen. Our third approach to the problem of controlling the ablated sample thickness is to incorporate a chromophore into the frozen solution, to localize energy deposition and ablation near the film surface and eliminate the dependence on the substrate as a chromophore, relaxing the requirements for controlled thin samples. However, to date, only sublimed frozen films have yielded good DNA mass spectra.

Figure 1 shows an early mass spectrum of a mixture of single-stranded DNA 8-mer and 20-mer. There are two characteristics of the mass spectrum worth noting. First, there is an intense cluster of low-mass peaks arising from the ablated copper substrate. We have not yet installed any deflectors to remove such low-mass peaks because our detection capability at higher mass does not appear to be impeded by this signal. Second, the 8-mer and 14-mer peaks are fairly clean and sit on a background that is entirely due to electronic noise effects. The sloping background arises from insufficiently suppressed pickup from the laser flashlamps which we have since eliminated. The absence of signal outside the peaks is in marked contrast to typical protein mass spectra obtained with UV irradiation in which the peaks ride on a continuous background arising from fragmentation and metastable decomposition. The evidence suggests that the protein molecular ions from UV irradiation 
are "hot" and barely survive the ablation process.

We have also made good progress in controlling the substrate surface conditions. In our pilot studies, success was achieved by allowing the copper substrate to corrode over a period of weeks by repeated applications of TE (tris-EDTA) buffer solutions as ablation studies proceeded. This produced a heterogeneously corroded surface which we believe contributed to the irreproducibility of the process -- i.e. it was necessary to search the sample to find an ice region of the correct thickness overlying a copper region with the right level of corrosion. We now corrode our copper substrates controllably by exposure for several minutes to acidified potassium permanganate solution. This produces a uniform black copper oxide surface which is both an excellent light absorber, and is also highly hydrophilic, so that aqueous samples can be spread uniformly over the surface.

Despite these improvements in control, the ablation/ionization process remains tantalizingly erratic. Good mass spectra are still only obtainable on small regions of the sample films, often after several hours of searching. Not infrequently, a sample is loaded from which it is impossible to obtain good mass spectra. There is clearly a variable which we do not yet recognize or control. With this caveat, we discuss below our progress towards DNA mixture analysis.

\section{II.b DNA test samples}

We have obtained from the ASU DNA synthesizer a set of single-stranded DNA samples of different lengths ranging from 8 to 60 bases to evaluate our technique. These fragments contain mixtures of the four DNA bases, and the sequences were specified to preclude either self-annealing or interfragment annealing.

To simulate a short Sanger fragment set, we constructed a 6-component test mixture with the following criteria: (a) the fragment sequences were specified to be noncomplementary so that they would not anneal to each other, and (b) the fragment masses were chosen so that no two fragments differed in base number by a factor of 2 . With these criteria, possible artifacts such as dimer formation and doubly-charged ion formation should be unambiguously detectable. The compositions of the six fragments are listed in Table 1.

Figures 2 and 3, obtained by David Schieltz, show the best mass spectra obtained to date with this test mixture. These mass spectra demonstrate many features required for successful sequence analysis. All six fragments from 8 to 60 bases yielded clean, wellresolved peaks. For the smaller fragments, peaks due to doubly-charged ions are seen, but these peak intensities are no more than - $20 \%$ of the main peaks. There is no evidence of cluster formation. The peak intensities for singly-charged ions differ by no more than a factor of 2 over the mass range, for an equimolar mixture. Uniform sensitivity is an essential attribute for error-free mixture analysis. Most significantly, the peaks lack the extended tails which characterize the "hot" protein peaks, and there are no detectable fragment peaks, nor a continuous fragment background. Such a clean mass spectrum is 
essential for accurate sequence ordering. The quality of the mass spectra suggests that the water matrix is acting as predicted to yield a "cool explosion" (see later discussion).

The resolving power (11-width, measured at $10 \%$ peak height) is - 50 at the highest mass ( $18,500 \mathrm{Da}$ ) (see inset: : For mixture analysis, this full-width resolving power is the required criterion. Resolving poners for protein mass spectra are typically quoted using the more relaxed criterion of full width at half maximum (FWHM) because the peaks contain substantial tails; the performance in Figs. 2 and 3 is c nsiderably better than any reported time-of-flight data for proteins at this mass. For comparison, Fig. 4 shows a mass spectrum of the protein lysozyme ( $\mathrm{MW}$ - 14,500 Da) obtained by UV laser ablation using an $\alpha$-cyano 4-hydroxycinnamic acid matrix. Note the extended tails on the peak, which sits on a continuous fragment background. Although the FWlAM resolving power for the protein peak ( ${ }^{-46}$ ) is only a factor of 2 worse than for the DNA data $\left({ }^{-} 80\right)$, the $10 \%$ resolving power is only - 13 for the protein peak compared to - 40 for the DNA 60-mer. Resolving power in sequence mixture analysis is directly related to the readable sequence length, so that the demonstrated resolving power in Figs 2 and 3 would allow read lengths no better than 50-60 bases. However, due to the difficulty of obtaining reproducible mass spectra (see below), we have not yet devoted any attention to improving the resolving power; significant improvement appears possible. These results are currently being written up for submission to Rapid Communications in Mass Spectrometry.

The data shown in Figs 2 and 3 comprise a 6-shot and a 9-shot average respectively in a small region of each frozen film. The total amount of sample loaded in each case was - 20 pmol of each DNA segment in a film smeared over $-2 \mathrm{~cm}^{2}$; however, the amount sampled to obtain the mass spectra was much smaller, $\sim 15-20 \mathrm{fmol}$. This is comparable with the sample sizes yielded by the Sanger sequencing method. However, we must emphasize that such performance is not the norm: the major part of each of these sample films produced no DNA molecular ion peaks, and spectra were more typically completely unobtainable from apparently similar sample films. Thus, despite the impressive promise of these mass spectra, we have continued to explore other approaches which might yield improved reproducibility.

\section{II.c Other matrices}

Ms. C-W Chou in my group has searched for materials which might act as ablation/ionization drivers for DNA under UV laser irradiation at $355 \mathrm{~nm}$ (the wavelength obtainable from our Nd-YAG laser by frequency tripling). She identified two key criteria: (i) the material should have a high extinction coefficient at $355 \mathrm{~nm}$, and (ii) the material should be as hydrophilic as possible. Other UV matrices which work for proteins, such as the $\alpha$-cyano 4-hydroxycinnamic acid matrix used to obtain Fig. 4 are only moderately hydrophilic, dissolve poorly in water, and appear to incorporate DNA strands very poorly into the crystal when dried. Ms. Chou suggested that anthranilic acid (2-amino benzoic acid) might be a UV-sensitive matrix suitable for DNA, because it begins to meet her two criteria. 
Fig. 5 shows the mass spectrum of a different test mixture assembled by $\mathrm{Ms}$. Chou ( sizes were $8,10,14,20,26,32$ bases) and obtained using an anthranilic acid matrix at 355 $\mathrm{nm}$. This mass spectrum compares favorably with others recently published using UV irradiation. (Note that a number of authors have obtained very good results with UV laser ablation of polythymidilic acids, but a more comprehensive study has recently shown that mass spectra of homopolymers of the other bases, and heteropolymers, are far more difficult, or impossible to obtain.) Ms. Chou has determined conditions under which spectra such as that of Fig. 5 are obtainable quite reproducibly, in contrast to our experience with ice film ablation.

Three features of Fig. 5 are noteworthy. First, the peaks ride on a fairly intense background signal. This is not an effect of saturation of the detector by low-mass signals - the mass spectrum was obtained at low power and the signal at low mass is not intense. Instead, this background must arise from fragmentation of the ablated DNA traversing the acceleration gap. Second, the resolved DNA peaks are quite broad, show tails to lower mass, and for the 8-, 10- and 14-mers fragment peaks begin to be resolved at the low mass side. This is more clearly apparent in the expanded view in Fig. 6 . Third, the peak intensity decreases abruptly beyond the $10-\mathrm{mer}$, in contrast to the ice film data in Figs. 2 and 3. The latter data show that this drop in intensity is not a detector problem, and must be related instead to extensive fragmentation of the higher-mass DNA segments. These observations again confirm that UV laser ablation is a far "hotter" process than ice film ablation, and that sufficient energy is coupled into the DNA molecules in the former approach to efficiently disintegrate larger molecules.

We are continuing to study this matrix, in the hope of finding ways to "cool" the process and improve the sensitivity and mass resolution. Approaches that we will try include using mixed matrices -- combining other UV or visible light absorbing materials with the anthranilic acid, or materials that might produce a more extensive gas plume and so aid the cooling process.

\section{Collector experiments}

By studying mass spectra alone, it is impossible to determine whether ablation, ionization or detection problems are to blame when good signals are not detected, and difficult to investigate mechanisms of ablation. For this reason, we have continued our collector studies. By collecting ablated material and analysing the mass distribution by electrophoresis, the ablation process can be studied in isolation. We look at two parameters: (i) the angular distribution of the collected deposit which gives information about the contribution of a supersonic jet expansion to the ablation process, and (ii) the fragmentation of the biomolecules which gives information on their energy content. Much of this work has been done with proteins rather than DNA because the electrophoresis is simpler (non-radioactive samples can be used), and both dry solid (dihydroxy-benzoic acid, DHB, a UV-absorbing matrix) and frozen (water matrix) samples have been studied. For both types of matrix, Ms. C-W Luo has observed forward-peaked angular distributions, 
consistent with the idea that ablation produces a vapor plume which undergoes a supersonic jet expansion. However, in the ice film case, the angular distribution of the ablated protein molecules is much more strongly forward-peaked, consistent with the idea that the larger vapor volume in the latter case produces an expansion with higher Mach number (which should give better cooling). Similar effects are seen in the fragment distribution; ice samples produce very little fragmentation of the protein lysozyme upon ablation, whereas the DHB matrix produces varying amounts of fragmentation. Consistent with our early results, the extent of fragmentation decreases as the power density is increased.

\section{Future plans: remainder of year 2 and year 3 .}

Our results to date lead to the following conclusions:

i) The aqueous matrix is capable of producing significantly higher-quality mass spectra than any of the solid/UV matrix materials have demonstrated to date. We believe this is due to two factors: first, compatibility of the matrix with the hydrophilic DNA analyte, and second, a much better-developed supersonic expansion which cools the ablated molecules and minimizes fragmentation.

ii) Ablation of intact molecules can routinely be achieved. Similarly detection of massive molecules is routinely possible in our system (this was demonstrated by obtaining, by UV laser ablation, reproducible mass spectra of massive proteins up to and beyond molecular weights of $100 \mathrm{kDa}$ ). The key difficulty appears to be that of ionization of the ablated molecules.

We had originally planned to build a second mass spectrometer in year 2 , dedicated to analytical studies, while the first instrument was retained for technique deveiopment. We have not yet begun to build this second instrument, because, as discussed above, we do not feel that we have sufficient control over the ablation/ionization process to decide on an optimum design. For example, one design decision is whether or not to incorporate an energy/time-focussing ion reflector to improve mass resolving power. Building such a reflector would be a major undertaking with our present approach because it would have to operate with voltages up to $30 \mathrm{kV}$. In protein work, others have found that such reflectors perform very poorly, because the "hot" protein molecular ions mostly decompose early in their flight, leaving little or no intact ion signal to be reflected; our DNA mass spectra suggest that this would be less of a problem because the ions appear "cooler", however, we do not yet have conclusive evidence on this point. The second design decision we cannot yet make concerns the choice of laser. We hope that there may be better solutions to the ionization problem than our present approach, and this will be a major focus of our work in the next several months.

Our work in the remainder of year 2 must focus on the ionization problem, together with the related problem of controllably making sample films which reproducibly yield mass spectra of the quality of Figs 2 and 3 . We will continue to work to controllably vary frozen 
film parameters to determine which are controlling the ionization process. In addition, however, we will use the understanding of the process that is developing from our combined collector and mass spectrometry studies to guide other approaches to our goal.

Our model of the process suggests that the vapor plume is quite cold (a few hundred kelvins) after expansion ceases, so that the molecules and atoms in the plume are overwhelmingly in their ground state -- i.e. neutral. Although we always see intense lowmass ions from the ablated substrate materia, to cbserve ions from the leading edge of the plume, where large molecules are expected to survive, we need some method of creating ions in this region. This may be what we do when we tune into the sodium atom resonance, producing excited sodium atoms which can then be ionized collisionally and attach to the DNA molecules, but we have not yt ascertained why this process does not occur routinely. So we are looking into other means of injecting energy, and producing ions, in the leading edge of the plume. It seems probable that this is what occurs in the UV ablation process - the plume begins to develop in the first few picoseconds of the laser pulse, and the ablated matrix molecules are capable of strong resonant absorption of the UV photons, so that energy continues to be injected into the plume as it develops during the several nanosecond laser pulse. We believe that it is for this reason that ablation with picosecond lasers is unsuccessful in producing large molecular protein ions. In our own case, the ablating laser is not absorbed by the majority species in the plume -- the water molecules - nor by the DNA. One approach to more consistent ionization is to change this.

In our earliest paper, we pointed out that the most logical laser for water ablation would operate at $2.9 \mu \mathrm{m}$, coupling energy directly into the $\mathrm{O}-\mathrm{H}$ stretch of water. At that time, we had no reason to believe that such a laser could also produce ions, but work since then by Franz Hillenkamp and Michael Karas of the University of Munster has shown, rather surprisingly, that such a laser (an Er-YAG laser was used) indeed produces protein molecular ions from a variety of matrices (including ice). The mechanism by which ions are produced by such low-energy ( $-0.3 \mathrm{eV}$ ) photons has not yet been elucidated, but we believe it likely that ionization results from absorption of multiple IR photons with intersystem crossings feeding this vibrational excitation into electronic excitation and eventually producing protons which attach to the ablated molecules. However, we suspect that the rather long pulse length of the Er-YAG laser ( $200 \mathrm{~ns}$ ) may have a deleterious effect on the mass spectrum, not only degrading mass resolution due to the long exciting pulse, but also, because the laser continues to pump energy into the ablated molecules at a time when expansion cooling is ceasing, producing unneeded fragmentation. We feel that a short pulse IR laser (10 ns or less) should be far more effective in producing cool molecular ions. Therefore, we have arranged to evaluate an optical parametric oscillator (OPO) which when pumped by our existing Nd-YAG will produce 5-8 ns pulses of IR, tuneable over a range from 1.5 to $4 \mu \mathrm{m}$. By tuning this laser into resonance with the water transition at $2.9 \mu \mathrm{m}$, we should achieve performance comparable to, or better than, that achieved by Hillenkamp and Karas. The extinction coefficient of ice or water at this wavelength is so great that ablation will take place at the outer surface of the sample film. Thus the need to achieve a critical sample thickness should be obviated. In addition, the 
tunability of the OPO laser opens other possibilities. For example, we helieve that a major advantage of ablating relatively thick films as in our present approach is the improved cooling from the better-developed supersonic water vapor jet expansion. By tuning the OPO laser off-resonance, it will be possible to vary the ablation depth, and thus the cooling efficiency of the vapor plume, and hopefully reach an optimum compromise between ionization efficiency and intact molecule survival. Because this approach is speculative at present, we have negotiated an evaluation period for the OPO system before making a purchase decision.

We will continue to pursue other approaches to ionization. One strategy that seems promising involves ion-pair dissociation. The DNA is now ablated as the free acid, or a sodium salt, and all evidence suggests that the plume temperature is such that ion-pair dissociation is negligible. This is consistent with the statistical mechanics of dissociation of a $2-3 \mathrm{eV}$ bond at temperatures below $1000 \mathrm{~K}$. One approach to ionization is to make this bond weaker, by making a DNA salt of a bulky organic base. In this way, the bond energy might be reduced to $-0.5-1 \mathrm{eV}$; however this is still not weak enough for significant dissociation at low temperature. We do not want to raise the temperature of the entire system, particularly the DNA molecules, because that will start to produce fragmentation. However, it is possible to incorporate in the organic base a chromophore which will selectively absorb photons from the ablating laser. Prof. Robert Levis at Wayne State University is working to achieve DNA ionization using multiphoton ionization of a chromophore covalently attached to the DNA molecule (Levis: personal communication). In such an approach the problem is to avoid either fragmenting the DNA molecule or splitting off the chromophore when the electronic excitation is internally converted to vibrational excitation. In contrast, our hope is that fragmentation will occur, leading to ionpair dissociation which leaves a negatively-charged DNA molecule. We are investigating this approach using rhodamine as a target chromophore and sublimed frozen films and room-temperature thin TE films containing DNA.

\section{Summary}

By pulsed laser ablation of frozen aqueous DNA solution films we have produced mass spectra of DNA mixtures which largely fulfil the simple requirement for DNA mixture analysis: one peak per DNA segment. The peaks are clean and free of the fragment or adduct tails which characteristically degrade mass spectra obtained by UV laser ablation using UV chromophore matrices. To date, our approach has been characterized by extremely pcor reproducibility; however the high quality of the mass spectra suggest that when better control of the ionization process is achieved, the use of an aqueous matrix offers an extremely promising approach to time-of-flight mass spectrometric sorting of DNA sequence mixtures. 


\section{Publications and presentations}

"Laser ablation of intact massive biomolecules", P. Williams, D. Schieltz, Cong-Wen Luo, R.M. Thomas and R.W. Nelson, in Laser Ablation: Mechanisms and Applications ed. by J.C. Miller and R.F. Haglund, Springer Verlag, Berlin 1991.

"Progress towards DNA sequence determination using laser ablation time-of-flight mass spectrometry", David M. Schieltz, Robert M. Thomas, Cong-Wen Luo, Chau-Wen Chou and Peter Williams, paper presented at 40th ASMS Conference on Mass Spectrometry and Allied Topics, Washington, D.C., June 1992.

$$
\text { Conf. pagens Cychend Dyparately }
$$


Table 1. DNA fragment set

$\begin{array}{lllllllllllll}\text { 8-mer } & \text { GAC } & \text { TGA } & \text { CT } & & & & & & & \\ \text { 14-mer } & \text { GAC } & \text { TGA } & \text { CTG } & \text { TGA } & \text { CA } & & & & & \\ \text { 20-mer } & \text { GAC } & \text { TGA } & \text { CTG } & \text { TGA } & \text { CTG } & \text { ACT } & \text { GT } & & & & \\ \text { 26-mer } & \text { GAC } & \text { TGA } & \text { CTG } & \text { TGA } & \text { CTG } & \text { ACT } & \text { GTG } & \text { ATC } & \text { GT } & & \\ \text { 32-mer } & \text { GAC } & \text { TGA } & \text { CTG } & \text { TGA } & \text { CTG } & \text { ACT } & \text { GTG } & \text { ACT } & \text { GTG } & \text { ACT } & \text { GT } \\ \text { 60-mer } & \text { GAT } & \text { CGA } & \text { TCG } & \text { TGA } & \text { TCG } & \text { ATC } & \text { GTG } & \text { ATC } & \text { GAT } & \text { CGT } & \text { GAT } \\ & \text { CGA } & \text { TCG } & \text { TGA } & \text { TCG } & \text { ATC } & \text { GTG } & \text { ATC } & \text { GAT } & \text { CGA } & & \\ & & & & & & & & & & & & \end{array}$




\section{Figure Captions}

Fig. 1. Early mass spectrum of a mixture of SS-DNA 8-mer and 20-mer ablated from a frozen aqueous solution film.

Fig. 2. Mass spectrum of equimolar DNA test mixture: 8, 14, 20, 26, 32, 60 nucleotides, ablated from frozen aqueous solution film. The sequences of the segments are listed in Table 1. The spectrum is an average of 9 laser shots. The inset shows an expanded view of the 60-mer region and the peak widths at $50 \%$ and $10 \%$ of maximum intensity. Amount of sample loaded was -20 pmol/DNA segment. Amount sampled to obtain this spectrum was $\sim 20$ fmol/DNA segment. Laser power $1.8 \mathrm{~mJ}$ into $100 \times 200 \mu \mathrm{m}$ spot; wavelength $578 \mathrm{~nm}$.

Fig. 3. Mass spectrum of equimolar DNA test mixture: 8, 14, 20, 26, 32, 60 nucleotides ablated from frozen aqueous solution film. The sequences of the segments are listed in Table 1. The spectrum is an average of 6 laser shots. The inset shows an expanded view of the 60-mer region and the peak widths at $50 \%$ and $10 \%$ of maximum intensity. Amount of sample loaded was $\sim 20$ pmol/DNA segment. Amount sampled to obtain this spectrum was 15 fmol/DNA segment. Laser power $2 \mathrm{~mJ}$ into $100 \times 200 \mu \mathrm{m}$ spot; wavelength $578 \mathrm{~nm}$.

Fig. 4. $\quad$ Mass spectrum of lysozyme (MW - 14,500 Da) ablated using $\alpha$-cyano 4hydroxycinnamic acid matrix. Laser pulse energy $<0.6 \mathrm{~mJ} /$ pulse into $2 \times 2$ mm spot. Laser wavelength: $355 \mathrm{~nm}$.

Fig. 5. Mass spectrum of equimolar DNA test mixture: 8, 10, 14, 20, 26, 32 nucleotides ablated using anthranilic acid matrix. Sequences are listed in Table 1 (the 10-mer has the 14-mer sequence, truncated after 10 bases). Laser power $<0.6 \mathrm{~mJ}$ into ${ }^{-} 2 \times 2 \mathrm{~mm}$ spot. Laser wavelength $355 \mathrm{~nm}$.

Fig. 6. $\quad$ Expanded view of mass spectrum of Fig. 5. 


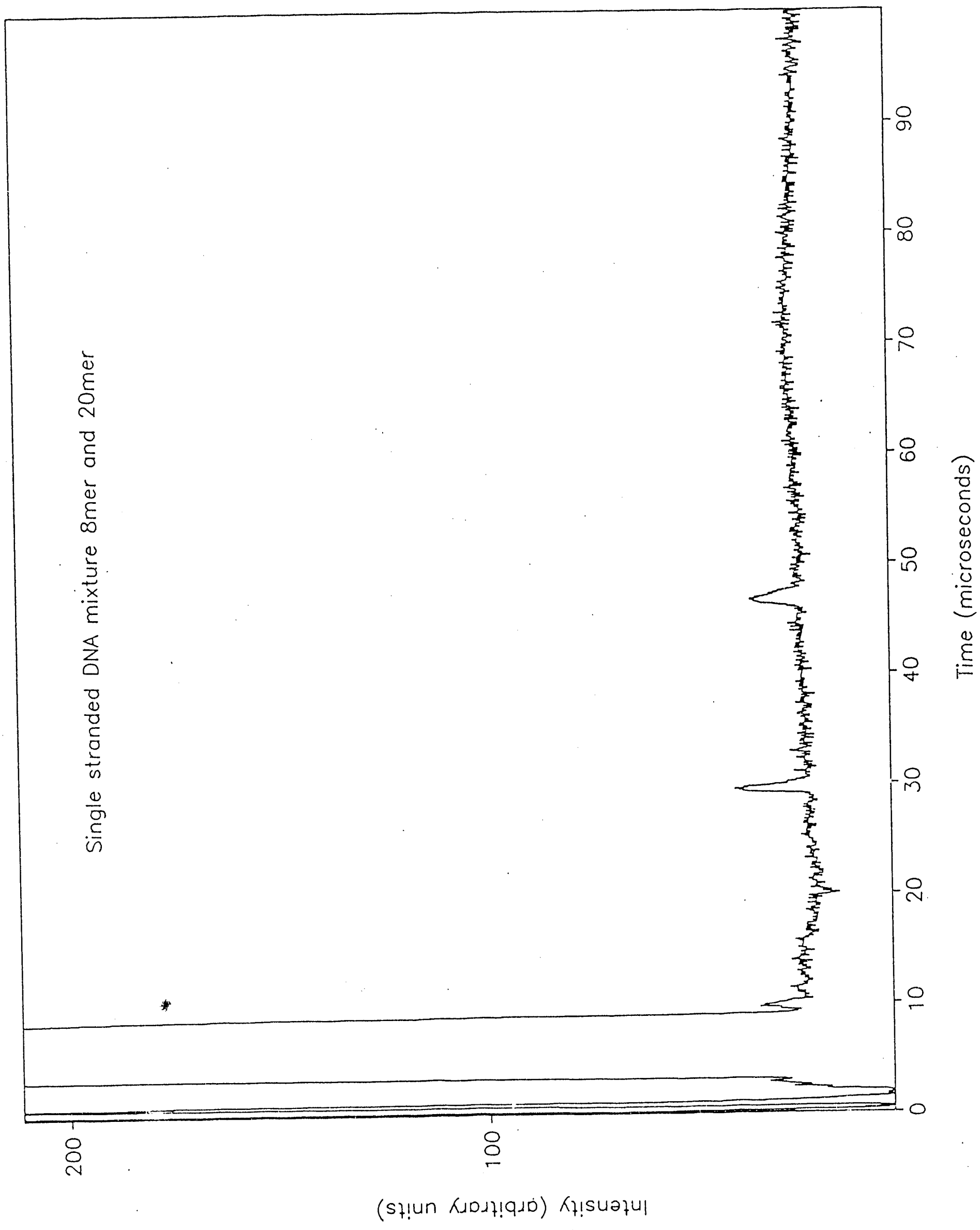


2 อxกธนุษ

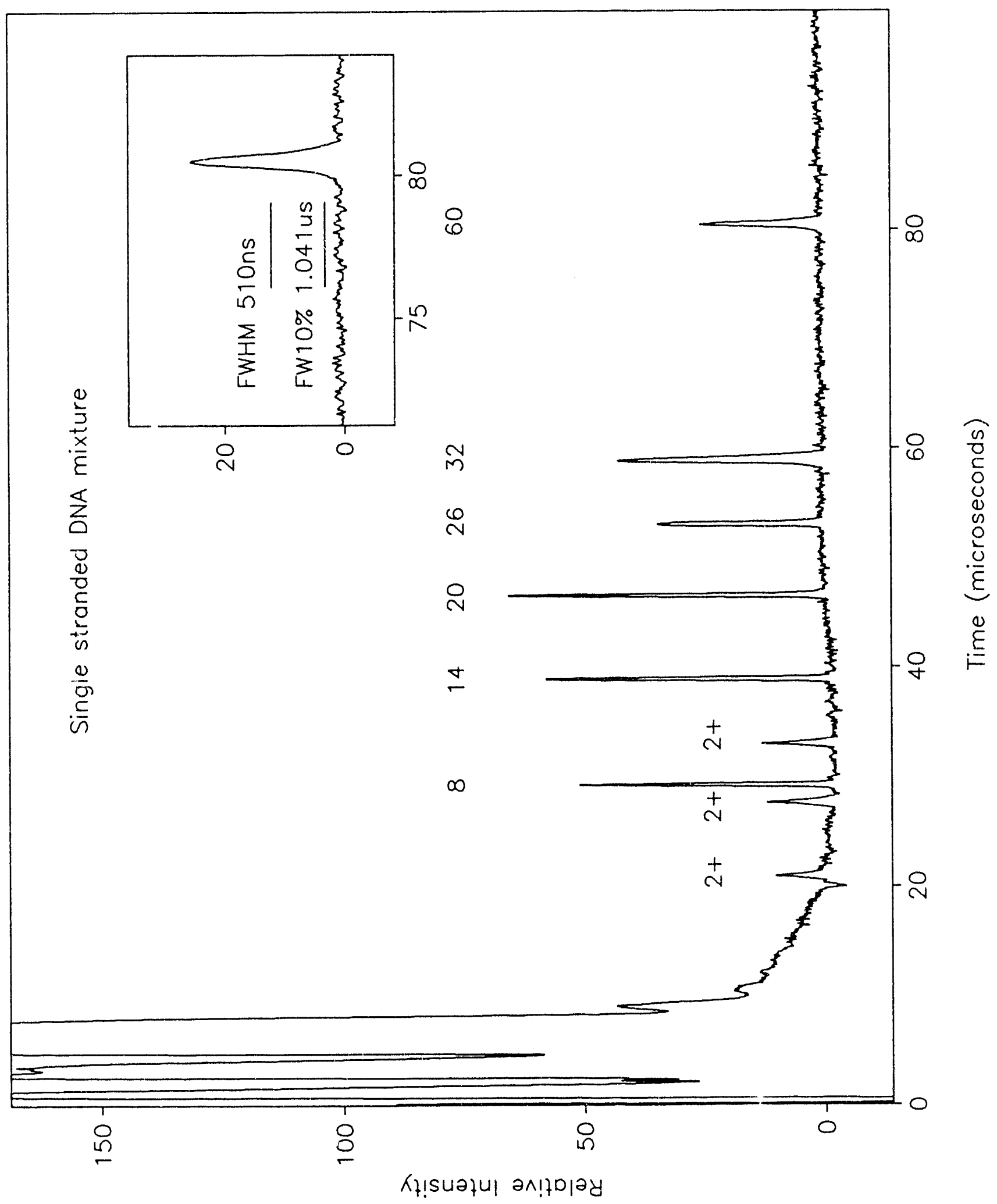


$\varepsilon$ วxnธเด

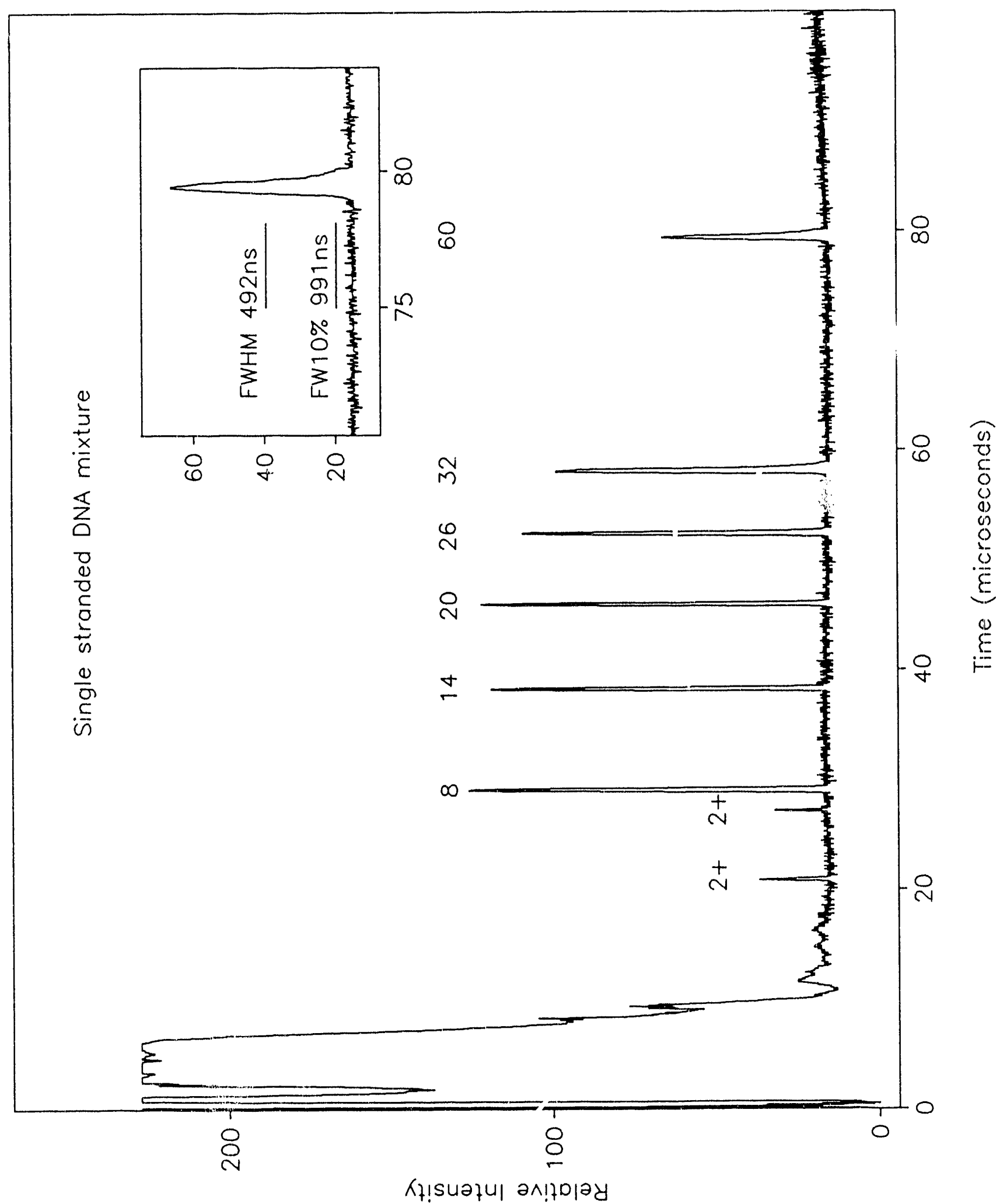




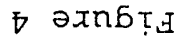

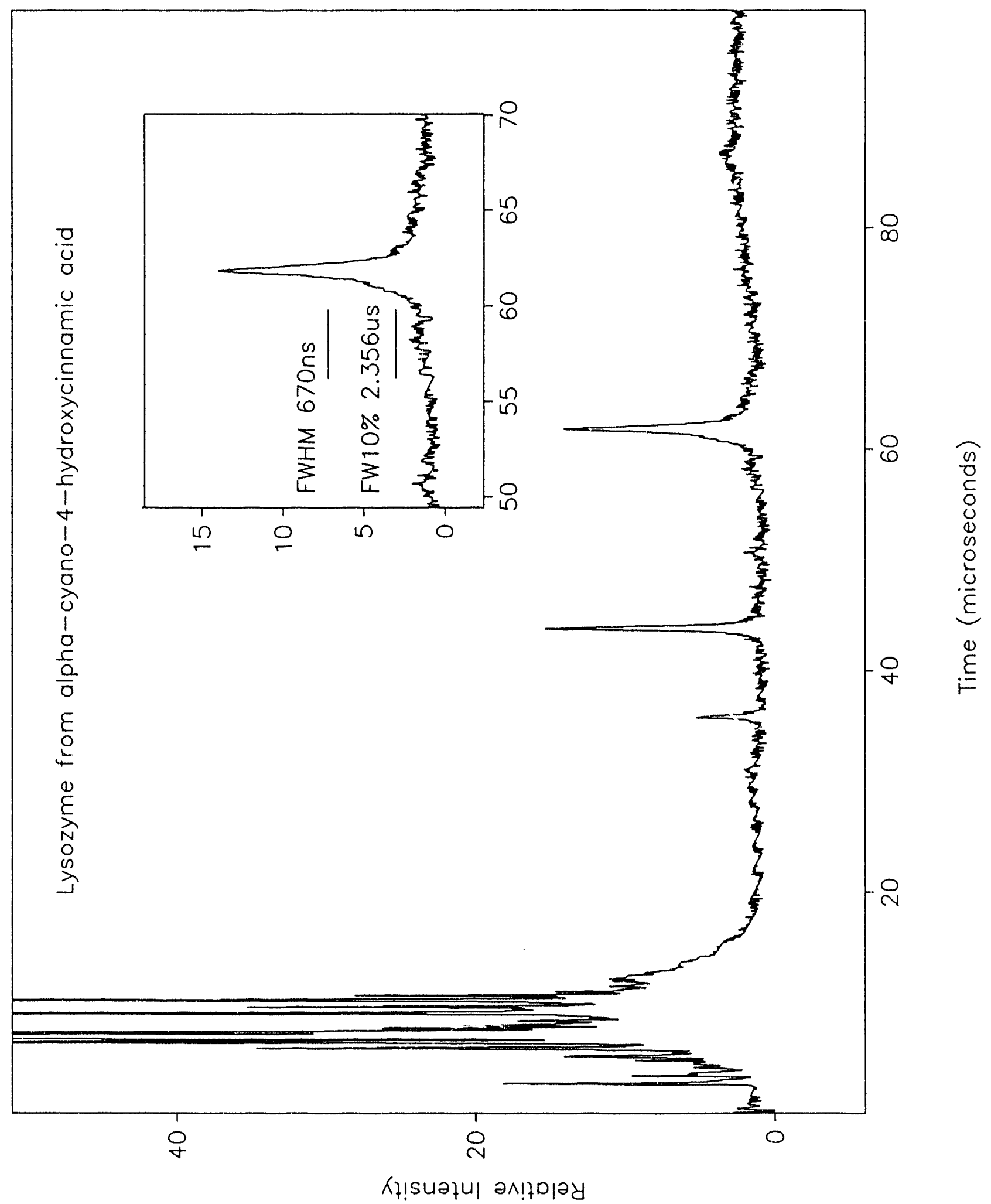


ร $ә \operatorname{xn} 6 T$

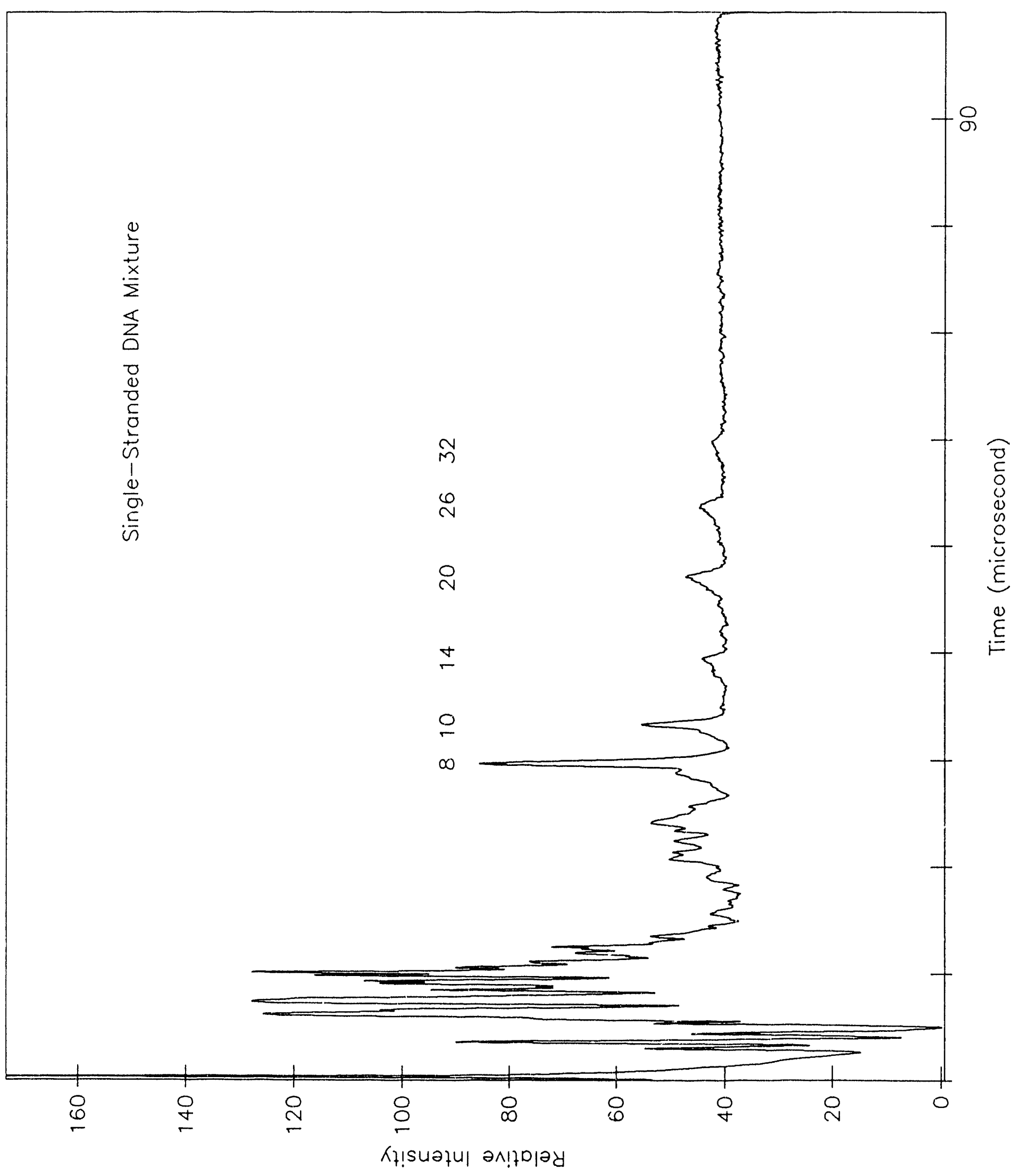


9 әxก6รู

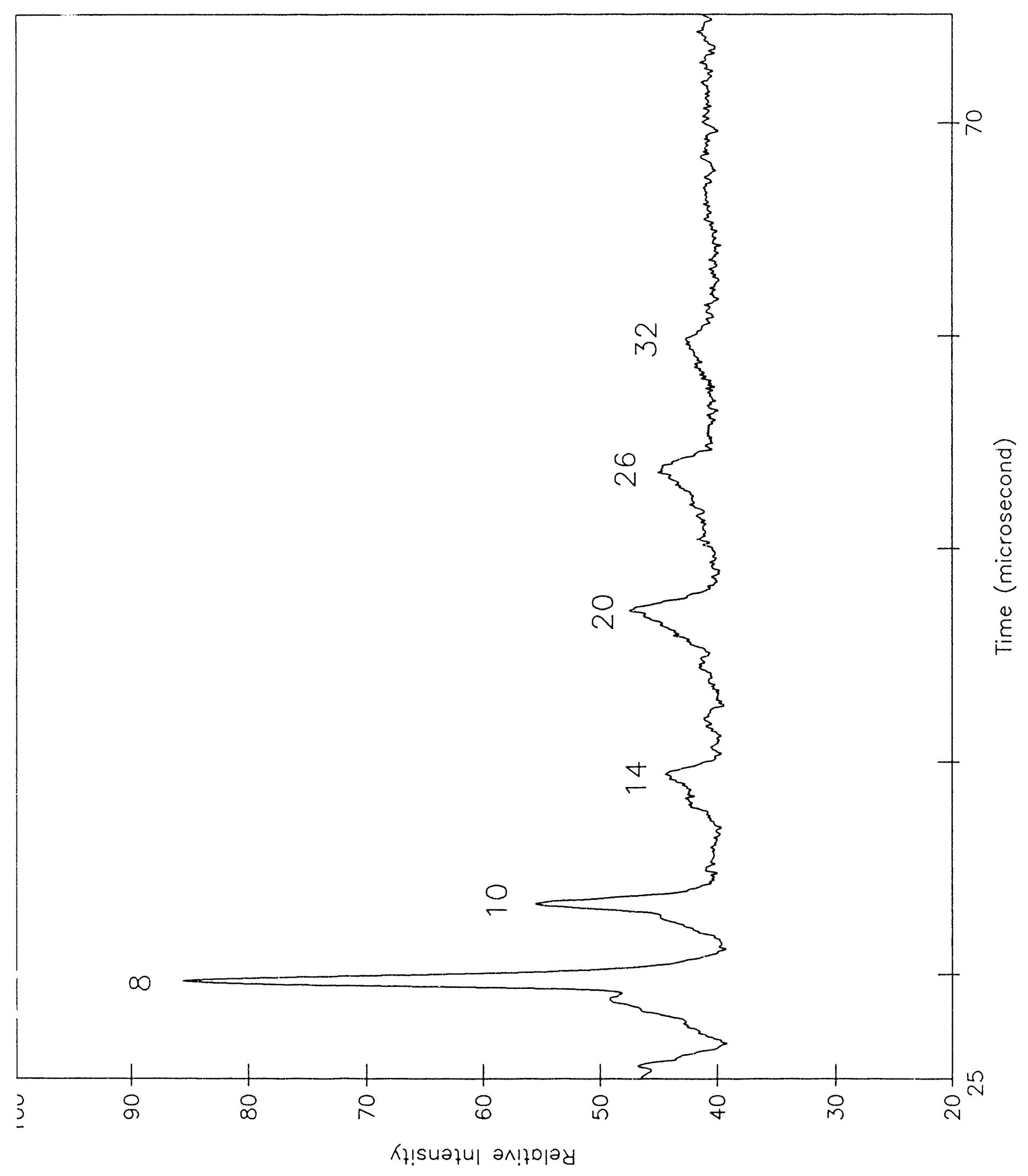



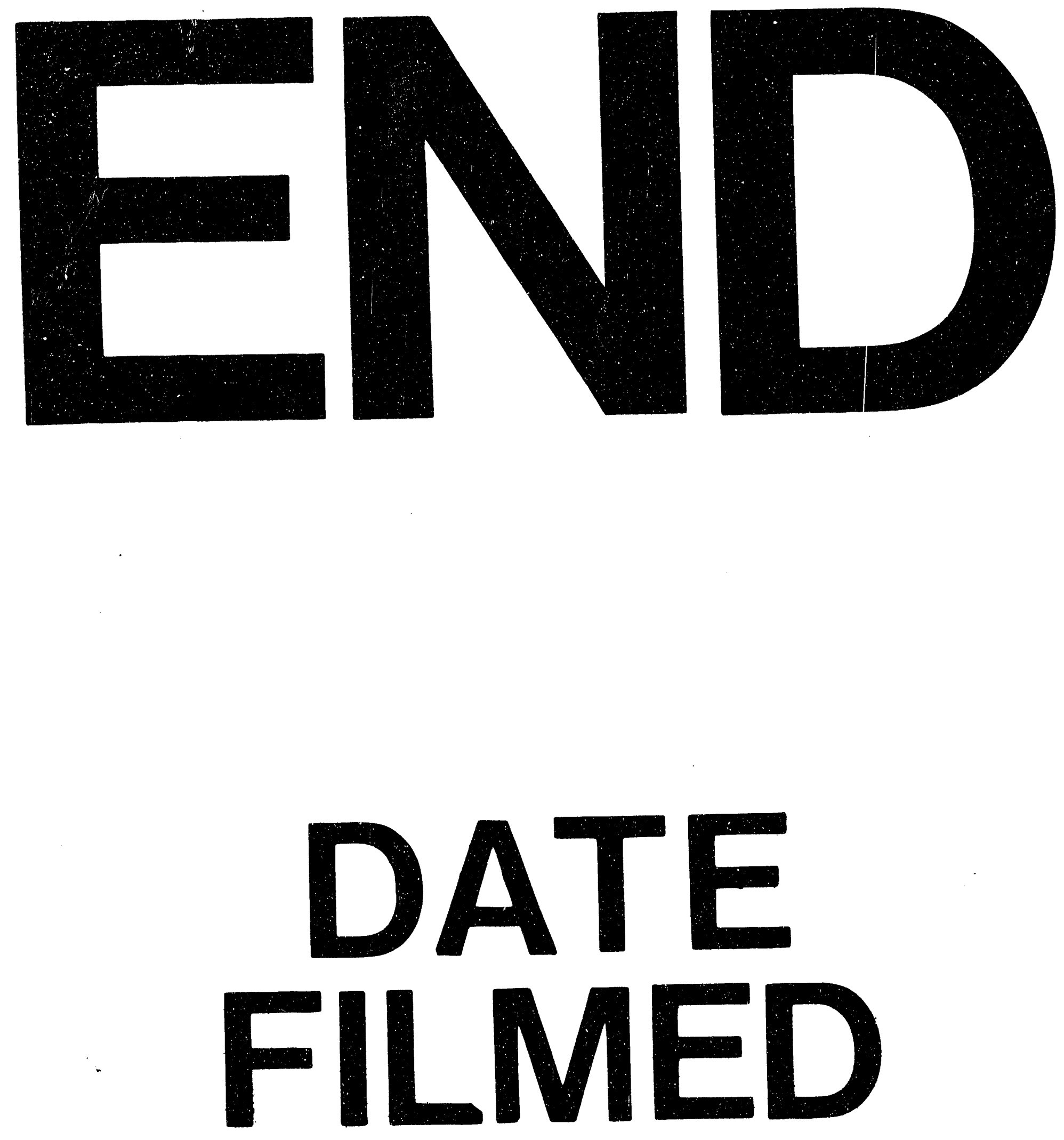

1

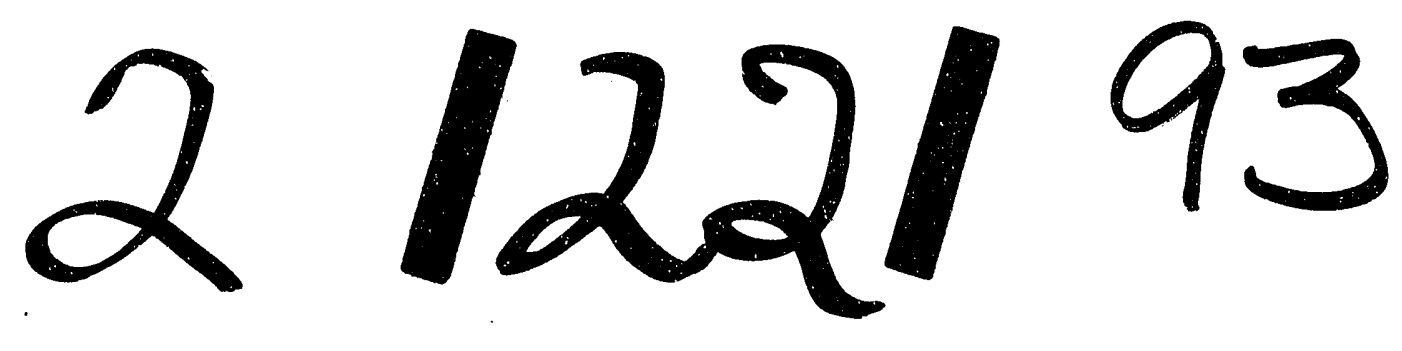


